\title{
Clinical significance of peripheral blood and tumor tissue lymphocyte subsets in cervical cancer patients
}

Yutuan $\mathrm{Wu}^{1,2+}$, Shuang $\mathrm{Ye}^{1,2+}$, Shyamal Goswami ${ }^{3}$, Xuan Pei ${ }^{1,2}$, Libing Xiang ${ }^{1,2}$, Xiaoming Zhang ${ }^{3}$ and Huijuan Yang ${ }^{1,2^{*}}$

\begin{abstract}
Background: Alterations in peripheral blood lymphocytes in cervical cancer have been reported, although conflicting views exist. The present study investigated the distributions of lymphocyte subsets in tumor tissue and peripheral blood samples from cervical cancer patients and precancerous lesion patients, and evaluated the correlations of lymphocyte subsets with clinicopathological and prognostic variables.

Methods: A total of 44 patients with stage IB1-IIA2 cervical cancer and 13 precancerous lesion patients were included. Lymphocytes were collected from the tumor tissue and the peripheral blood, and isolated by Lymphoprep density gradient centrifugation. The percentages of lymphocyte subsets were quantified by flow cytometry analysis, and the differences between lymphocyte subsets in the tumor tissue and peripheral blood were compared by SPSS. In addition, the relationships between lymphocyte subsets and clinicopathological and prognostic variables were analyzed.

Results: Our results revealed that the amount of total T lymphocytes, CD8+ T cells, granulocytes, pDCs, CD16+ monocytes and CD56 high NK cells were significantly higher in the tumor tissue than in the peripheral blood in the cervical cancer patients, while those of CD4+ T cells, CD4+/CD8+ cell ratio, rdT cells, BDCA1+ mDCs, total monocytes, CD14+ monocytes, NK cells and CD56 ${ }^{\text {low }}$ NK cells exhibited the opposite trend $(p<0.05)$. The levels of total $\mathrm{pDCs}$ and BDCA1+ mDCs in the peripheral blood were significantly lower in the cervical cancer patients than in the precancerous lesion patients, while the proportion of CD16+ monocytes was elevated $(p<0.05)$. In addition, some lymphocyte subsets, especially CD4+ cells and CD8+ cells, and the CD4+/CD8+ cell ratio were closely associated with clinicopathological and prognostic parameters.

Conclusions: These results suggested that distinct alterations in infiltrating lymphocyte subsets occurred in the tumor and were associated with clinicopathological and prognostic parameters. Systemic impairment of the immune system may occur in the antitumor response of cervical cancer patients.
\end{abstract}

Keywords: Cervical cancer, Lymphocyte subsets, Tumor immunology, Clinicopathological variables, Prognosis

\footnotetext{
* Correspondence: huijuanyang@hotmail.com

${ }^{\dagger}$ Yutuan Wu and Shuang Ye contributed equally to this work.

'Department of Gynecological Oncology, Fudan University Shanghai Cancer

Center, Fudan University, Shanghai, China

${ }^{2}$ Department of Oncology, Shanghai Medical College, Fudan University,

Shanghai 200032, China

Full list of author information is available at the end of the article
}

(c) The Author(s). 2020 Open Access This article is distributed under the terms of the Creative Commons Attribution 4.0 International License (http://creativecommons.org/licenses/by/4.0/), which permits unrestricted use, distribution, and reproduction in any medium, provided you give appropriate credit to the original author(s) and the source, provide a link to the Creative Commons license, and indicate if changes were made. The Creative Commons Public Domain Dedication waiver (http://creativecommons.org/publicdomain/zero/1.0/) applies to the data made available in this article, unless otherwise stated. 


\section{Background}

Cervical cancer is still the leading gynecological malignancy in China, with 98,900 new cases diagnosed in 2015 and over 30,500 deaths [1]. It has been widely accepted that persistent high-risk human papillomavirus (HPV) infection is the major risk factor for cervical cancer $[2,3]$. Furthermore, the local immune response is regarded as a key determinant in cervical carcinogenesis after HPV infection [4]. Evidence suggests that cellmediated immunity may be especially important in HPV-related malignancies, including cervical cancer [5].

Quite a few publications have tried to investigate the immunological status in cervical cancer and/or precancerous lesions [5-15]. Colleagues from France characterized $\mathrm{T}$ cells (CD4+, $\mathrm{CD} 8+, \mathrm{CD} 45 \mathrm{RO}+)$ by immunohistochemistry in high-risk HPV-infected cervical cancers and premalignant lesions [8]. Using flow cytometry, Das et al. assessed peripheral blood lymphocyte subpopulations in cervical cancer patients among Indian women, including only five parameters (CD4+ helper $\mathrm{T}$ cells, CD8+ cytotoxic $\mathrm{T}$ cells, CD16+ cells, CD19+ cells and CD56+ cells) [5]. Another interesting publication specifically described systemic $\mathrm{T}$ cell responses to HPV 16 and HPV 18 proteins [11]. In our previous work (a manuscript published in Chinese), we evaluated $\mathrm{CD} 4^{+} \mathrm{CD} 25^{\text {high }} \mathrm{CD} 127^{\text {low }}$ regulatory $\mathrm{T}$ cells in the peripheral blood of cervical cancer and precursor lesion patients [16]. We found that regulatory $\mathrm{T}$ cells were increased in the patients with cervical intraepithelial neoplasia or cancer [16].

The current study had several objectives. First, this study aimed to describe the immune cell landscape in cervical cancer patients relatively comprehensively, including the landscapes in the peripheral blood, tumor tissue and precancerous lesions. Second, we sought to evaluate possible correlations between lymphocyte subsets and clinicopathological variables. Last, the prognostic implications of immune cell levels were also investigated.

\section{Methods}

\section{Patient selection and sample collection}

We obtained approval from the institutional review board at Fudan University Shanghai Cancer Center (SCCIRB-090371-2). Cervical cancer patients who were scheduled for radical hysterectomy were enrolled in this study between April and November 2013. The included patients were required to fulfill the following criteria: 1) preoperative diagnosis of squamous cell carcinoma or adenocarcinoma of the cervix; 2) International Federation of Gynecology and Obstetrics (FIGO) stage IB1IIA2 disease, with visible gross tumor; 3) no receipt of preoperative treatment including chemotherapy and radiotherapy; and 4) provision of informed content.
Patients with a medical history of autoimmune diseases were excluded. All patients were human immunodeficiency virus (HIV) negative. Clinicopathological information was retrospectively abstracted from patient electronic medical records by a well-trained gynecological oncologist.

Peripheral blood was collected on the day before surgery. Attending surgeons were responsible for choosing and dissecting highly suspicious fresh tumor tissue for further analysis. We also collected peripheral blood samples from 13 patients who received cervical conization for precancerous lesions during the same period.

All the cervical cancer patients at our institution were required to have regular follow-up visits after surgery. Progression-free survival (PFS) was defined as the time interval from the date of primary surgery to the date of disease progression or recurrence.

\section{Cell isolation from tumor tissue}

Freshly resected cervical tissue samples from primary tumors were transferred to the laboratory immediately after surgery and processed within four hours. The fresh tumor tissue was gently minced into 3 to $4 \mathrm{~mm}$ cubes using a scalpel, followed by enzymatic digestion in a cocktail of collagenase type IV $(1 \mathrm{mg} / \mathrm{mL}$, Gibco, Carlsbad, CA, USA), and DNase I (100 $\mu \mathrm{g} / \mathrm{mL}$, Sigma, USA) in RPMI 1640 medium (HyClone, Utah, USA) and then processed into single-cell suspensions using a gentleMACS Octo Dissociator with Heater (Miltenyi, Bergisch Gladbach, Germany) in the preprogrammed human tumor mode-II at $37^{\circ} \mathrm{C}$ for 60 min. Released cells were collected and filtered through $70 \mu \mathrm{m}$ nylon cell strainers (BD, CA, USA) and processed for density gradient centrifugation to isolate leukocytes.

\section{Peripheral blood mononuclear cell (PBMC) isolation and staining}

Peripheral blood mononuclear cells (PBMCs) were collected by Lymphoprep (Axis-Shield) density gradient centrifugation of venous peripheral blood from patients treated with the anticoagulant K3ethylenediaminetetraacetic acid (EDTA, BD Cat No. 6457). For whole-blood staining, samples underwent erythrolysis with an ammonium-buffered solution (BD FACS Lysing Solution, $10 \mathrm{~min}$ at RT). The cells were resuspended in ice-cold Fluorescence Activated Cell Sorter (FACS) buffer containing $1 \mathrm{x}$ phosphate-buffered saline (PBS) $\left(\mathrm{Ca} / \mathrm{Mg}^{++}\right.$free, $\left.\mathrm{pH} 7.2\right), 2.5 \mathrm{mM}$ EDTA, $25 \mathrm{mM}$ HEPES and 1\% FBS. Samples were assessed for the expression of 18 markers and stained with Zombie Yellow (BioLegend), which was used to discriminate live and dead cells according to the manufacturers' recommendation, prior to surface staining. For surface staining, filtered single cells were incubated for $30 \mathrm{~min}$ on ice with 
an Fc receptor binding inhibitor (eBioscience) diluted 1/ 10 in PBS. The cells were then incubated in FACS buffer with combinations of fluorochrome-conjugated or biotinylated antibodies for $15 \mathrm{~min}$ in the dark at room temperature. The cells were centrifuged, and the pellets were recovered, fixed in a $1 \%$ paraformaldehyde solution and filtered through a $70 \mu \mathrm{m}$ mesh strainer to remove any small clumps immediately before flow cytometry analysis.

\section{Flow cytometry analysis}

The antibodies and clones used in the study are presented in the Additional file 1. Samples were acquired using a BD LSRFortessa flow cytometer (BD Biosciences) equipped with five lasers. Before running samples, the alignment of the instrument was checked daily using fluorescent microbeads; also, the accuracy of the volumetric apparatus was addressed by using commercial fluorescent beads having predetermined acceptable ranges for validation. Compensation was performed according to the cytometer manufacturer's instructions, allowing the use of an off-line procedure by applying automated electronic algorithms and preset templates; by using biparametric logarithmic dot plots, gate-specific tubes and single-tube data analysis; and by optimizing the FSC threshold and fluorochrome voltage as set-up parameters. All the data were analyzed with FlowJo software 9.7.3 (TreeStar, Inc. Ashland, OR).

\section{Statistical analyses}

Statistical Package for Social Science (SPSS) statistical software (Version 20.0, SPSS, Inc., Chicago, IL, USA) was used for analyses. Continuous data are presented as the median (range), and categorical data are presented as proportions. Parametric Student's $t$ tests were employed to evaluate continuous variables. The associations between different variables were evaluated using univariate and multivariate logistic regression analysis, and hazard ratios (HRs) with 95\% confidence intervals (CIs) were calculated. All of the $P$ values reported are two sided, and a value of $P<0.05$ was considered statistically significant.

\section{Results}

\section{Demographics}

Figure 1 shows the schematic of patient enrollment in the study. A total of 44 patients were ultimately included, and 78 samples were available for analysis. Among the patients, 34 had paired blood-tissue samples. Clinical and pathological information for the entire cohort is presented in Table 1 . The majority of the patients had a squamous histology $(n=40,90.9 \%)$. Deep stromal invasion ( $\geq 1 / 2$ depth), lymph node metastasis and positive lymphovascular invasion occurred in 70.5, 40.9 and

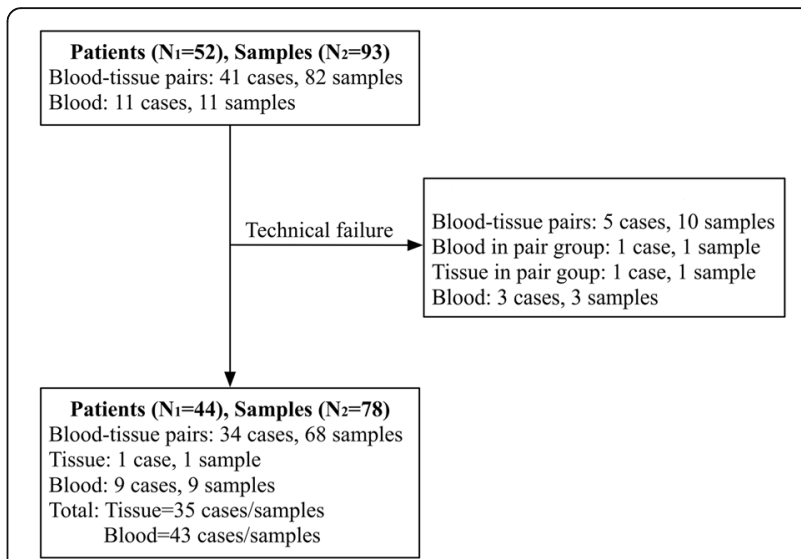

Fig. 1 Schematic of patients included in the present study

$47.7 \%$ of the patients, respectively. With a median follow-up time of 41 months, 36 patients $(81.8 \%)$ were alive with no evidence of disease, while 8 patients (18.2\%) had experienced disease recurrence.

\section{Lymphocyte subpopulations in the peripheral blood versus tumor tissue}

We collected paired blood-tissue samples from 34 patients, and compared different lymphocyte subsets in the tumor tissue versus the peripheral blood. The distributions of the lymphocyte subsets in the peripheral blood compared with those in the tumor tissue are presented in Table 2.

Table 1 Patient characteristics $(n=44)$

\begin{tabular}{ll}
\hline Median age (range), years & $47(23-70)$ \\
\hline FIGO stage & $15(34.1 \%)$ \\
IB1 (\%) & $8(18.2 \%)$ \\
IB2 (\%) & $11(25.0 \%)$ \\
IIA1 (\%) & $10(22.7 \%)$ \\
IIA2 (\%) & \\
Histology & $40(90.9 \%)$ \\
Squamous carcinoma (\%) & $4(9.1 \%)$ \\
Adenocarcinoma (\%) & $31(70.5 \%)$ \\
Stromal invasion $\geq 1 / 2$ depth (\%) & $18(40.9 \%)$ \\
Lymph node metastasis (\%) & $21(47.7 \%)$ \\
Positive lymphvascular invasion (\%) & $41(2-48)$ \\
Median follow up (range), months & \\
Disease status at last follow-up & $36(81.8 \%)$ \\
Alive, no evidence of disease (\%) & $5(11.4 \%)$ \\
Alive, with disease (\%) & $3(6.8 \%)$ \\
Dead with disease (\%)
\end{tabular}

Abbreviations: FIGO International Federation of Gynecology and Obstetrics 
Table 2 Lymphocyte subsets in peripheral blood versus tumor tissue $(n=34)$

\begin{tabular}{llll}
\hline & Blood & Tissue & $P$ value* \\
\hline Gran \%CD45 & $3.24 \pm 5.72$ & $10.55 \pm 12.71$ & 0.002 \\
T \%MNC & $55.59 \pm 12.03$ & $68.58 \pm 17.81$ & $<0.001$ \\
CD4\%T & $54.04 \pm 13.18$ & $45.85 \pm 14.49$ & 0.013 \\
CD8\%T & $32.37 \pm 11.22$ & $44.92 \pm 13.98$ & $<0.001$ \\
CD4:CD8 & $1.94 \pm 0.94$ & $1.22 \pm 0.74$ & 0.001 \\
rdT \%T & $7.53 \pm 4.57$ & $2.68 \pm 1.77$ & 0.001 \\
B \% MNC & $5.93 \pm 2.92$ & $5.00 \pm 3.44$ & $0.302^{\#}$ \\
pDC \% MNC & $0.24 \pm 0.13$ & $0.67 \pm 0.79$ & 0.003 \\
CD11c+ \% pDC & $8.13 \pm 5.08$ & $5.90 \pm 4.74$ & $0.109^{\#}$ \\
Baso \% MNC & $0.83 \pm 0.50$ & $0.09 \pm 0.16$ & $<0.001$ \\
BDCA1+ mDC \% MNC & $0.37 \pm 0.17$ & $0.11 \pm 0.14$ & $<0.001$ \\
BDCA3+ mDC \% MNC & $0.02 \pm 0.04$ & $0.02 \pm 0.06$ & $1.000^{\#}$ \\
Mono \% MNC & $16.69 \pm 9.38$ & $8.66 \pm 8.29$ & $<0.001$ \\
CD14+ Mo \% MNC & $14.76 \pm 7.03$ & $2.28 \pm 2.34$ & $<0.001$ \\
CD16+ Mo \% MNC & $3.27 \pm 2.25$ & $5.85 \pm 5.75$ & 0.036 \\
CD16+ \%Mono & $18.45 \pm 7.79$ & $71.62 \pm 13.20$ & $<0.001$ \\
NK \%MNC & $17.27 \pm 9.83$ & $5.88 \pm 12.63$ & $<0.001$ \\
CD56hi NK \% MNC & $0.33 \pm 0.16$ & $4.38 \pm 11.71$ & $0.079^{\#}$ \\
CD56lo NK \% MNC & $17.66 \pm 9.97$ & $1.24 \pm 1.94$ & $<0.001$ \\
CD56hi \%NK & $2.42 \pm 1.81$ & $65.31 \pm 23.69$ & $<0.001$ \\
MDSC \% CD33hi & $1.85 \pm 3.50$ & $2.39 \pm 3.83$ & $0.592^{\#}$ \\
DR- \%14 + APC & $0.41 \pm 1.50$ & $18.13 \pm 18.68$ & $<0.001$ \\
DR- \%16+ APC & $0.67 \pm 1.08$ & $1.64 \pm 2.96$ & $0.059^{\#}$ \\
\hline Pairdt & & &
\end{tabular}

*Paired t test

${ }^{\#} P$ values with no statistical significance were denoted

Abbreviations: Gran Granulocyte, MNC Mononuclear Cell, DC Dendritic Cell, pDC Plasmacytoid Dendritic Cell, $m D C$ Myeloid Dendritic Cell, NK Natural Killer, hi high, lo low, MDSC Myeloid-Derived Suppressor Cell, APC Antigen Presenting Cell, Baso Basophilic granulocyte, Mono Monocyte;

\section{Distributions of $T$ lymphocytes, granulocytes and B lymphocytes}

The amount of total $\mathrm{T}$ lymphocytes and granulocytes were significantly higher in the tumor tissue than in the peripheral blood $(p<0.05)$. However, a significant decrease in the $\mathrm{T}$ helper lymphocyte (CD4+ cell) population in the tumor tissue was observed compared to that in the peripheral blood $(p<0.05)$, while the level of $\mathrm{T}$ suppressor lymphocytes (CD8+ cells) was found to be significantly increased in the tumor tissue compared to the peripheral blood $(p<0.05)$. The CD4+/CD8+ cell ratio was significantly lower in the tumor tissue than in the peripheral blood $(p<0.05)$. Furthermore, the proportion of rdT cells, which play an important role in the function of innate immunity, was significantly lower in the tumor tissue than in the peripheral blood $(p<0.05)$. All of these results indicated that antitumor immunity was suppressed in the tumor tissue. However, no significant difference was observed for B lymphocytes when comparing the tumor tissue with the peripheral blood $(p>0.05)$.

\section{Distributions of dendritic cells and basophilic granulocytes} Dendritic cells (DCs) shape both innate and adaptive immune responses, including antitumor immune responses. There are two main types of DCs that are distinguished on the basis of different sources of differentiation, plasmacytoid dendritic cells (pDCs) and myeloid dendritic cells (mDCs). The proportion of pDCs, which are considered to induce more $\mathrm{T}$ regulatory cells (Tregs) and to promote immune escape by tumor cells, was significantly higher in the tumor tissue than in the peripheral blood $(p<0.05)$. However, CD11c $+\mathrm{pDCs}$, a major kind of pDCs, showed no significant difference between the tumor tissue and peripheral blood. Recently, two subsets of naturally occurring human blood-derived mDCs, BDCA1+/CD1c + mDCs and BDCA3+/CD141+ $\mathrm{mDCs}$, have been described. Our results indicated that the level of BDCA1+ mDCs was significantly lower in the tumor tissue than in the peripheral blood $(p<0.05)$. However, there was no significant difference in BDCA3+ $\mathrm{mDCs}$ between the tumor tissue and peripheral blood. In addition, the proportion of basophilic (Baso) granulocytes was significantly lower in the tumor tissue than in the peripheral blood $(p<0.05)$.

\section{Distributions of monocytes, NK cells and myeloid-derived suppressor cells}

Total monocyte and CD14+ monocyte frequencies were significantly decreased in the tumor tissue compared to the peripheral blood $(p<0.05)$. In contrast, the CD16+ monocyte frequency was significantly higher in the tumor tissue than in the peripheral blood $(p<0.05)$. Moreover, the subsets of natural killer cells (NK cells), which are believed to have the function of immune surveillance, were significantly decreased in the tumor tissue compared to the peripheral blood $(p<0.05)$. The CD56 $6^{\text {high }} \mathrm{NK}$ cell proportion was significantly higher in the tumor tissue than in the peripheral blood $(p<0.05)$, but the CD56 ${ }^{\text {low }}$ NK cell proportion exhibited the opposite trend $(p<0.05)$. Furthermore, myeloid-derived suppressor cells (MDSCs) showed no significant difference between the tumor tissue and peripheral blood.

\section{Lymphocyte subpopulations in the peripheral blood: cervical cancer versus precancerous lesions}

We detected the levels of different lymphocyte subpopulations in peripheral blood samples from cervical cancer patients and precancerous lesion patients. The results showed that the percentages of total pDCs and BDCA1+ mDCs were significantly lower in the cervical cancer patients than in the precancerous lesion patients; meanwhile, the CD16+ monocyte and CD56 ${ }^{\text {low }}$ NK cell 
proportions were higher in the cervical cancer patients than in the precancerous lesion patients, and the differences were significant between the cervical cancer patients and precancerous lesion patients $(p<0.05)$ (Table 3). However, other lymphocyte subpopulations were not significantly different between the cervical cancer patients and precancerous lesion patients $(p>0.05)$ (Table 3).

\section{Associations of lymphocyte subsets with clinicopathological parameters}

We correlated the distributions of lymphocyte subsets with clinicopathological parameters, such as age, FIGO stage, histology, tumor size, stromal invasion status, lymph node (LN) metastasis status and lymphovascular space invasion (LVSI) status.

Age, tumor size and LVSI status are closely related to the distributions of lymphocytes in the peripheral blood In the analysis of the peripheral blood, the results showed that the CD16+ monocyte proportion was significantly lower in the age $\leq 47$ group than in the age $>$ 47 group $(p<0.05)$ (Table 4$)$. Additionally, total $\mathrm{T}$ cell and CD56 $6^{\text {high }}$ NK cell proportions were significantly lower in the tumor size $\leq 4 \mathrm{~cm}$ group than in the tumor size $>4 \mathrm{~cm}$ group, while the BDCA3+ $\mathrm{mDC}$, total $\mathrm{NK}$ cell and CD56 ${ }^{\text {low }}$ NK cell proportions exhibited the opposite trend $(p<0.05)$ (Table 4$)$. The CD11c $+\mathrm{pDC}$ proportion was significantly lower in the LVSI-positive patients than in the LVSI-negative patients $(p<0.05)$ (Table 4). In addition, there were no other significant differences between clinicopathological parameters and lymphocyte subsets in the peripheral blood.

\section{Age, tumor size, LN metastasis status and LVSI status are closely related to the distributions of lymphocytes in tumor tissue}

In tumor tissue, the rdT cell proportion was significantly higher in the age $\leq 47$ group than in the age $>47$ group $(p<0.05)$ (Table 4). The levels of CD8+ cells, $\mathrm{pDCs}$, total monocytes, CD14+ monocytes and CD 16+ monocytes were significantly higher in the tumor size $>4 \mathrm{~cm}$ group than in the tumor size $\leq 4 \mathrm{~cm}$ group $(p<0.05)$ (Table 4$)$. Our results indicated that tumor size was closely related to the distributions of some lymphocyte subsets in both the peripheral blood and the tumor tissue. LN metastasis was correlated with significant reductions in the level of CD4+ cells and the CD4+/CD8+ cell ratio but also with increased levels of CD8+ cells and CD16+ monocytes $(p<0.05)$ (Table 4). Contradictorily, LVSI was correlated with a significantly reduced level of CD8+ cells but also with an increased level of CD4+ cells and an elevated

Table 3 Lymphocyte subsets in peripheral blood: cervical cancer versus precancerous lesion

\begin{tabular}{|c|c|c|c|}
\hline & Cancer $(n=43)$ & Precancerous lesion $(n=13)$ & $P$ value $^{*}$ \\
\hline Gran \%CD45 & $3.05 \pm 5.29$ & $4.17 \pm 6.17$ & 0.522 \\
\hline T\%MNC & $57.41 \pm 12.79$ & $59.58 \pm 9.54$ & 0.575 \\
\hline rdT \% & $7.30 \pm 4.45$ & $4.83 \pm 2.22$ & 0.067 \\
\hline B \%MNC & $6.05 \pm 3.11$ & $6.48 \pm 2.48$ & 0.644 \\
\hline $\mathrm{pDC} \% \mathrm{MNC}$ & $0.25 \pm 0.13$ & $0.35 \pm 0.16$ & $0.022^{\#}$ \\
\hline$C D 11 c+\% p D C$ & $7.62 \pm 5.03$ & $6.18 \pm 1.97$ & 0.386 \\
\hline Baso \% MNC & $0.98 \pm 0.71$ & $1.19 \pm 0.60$ & 0.334 \\
\hline $\mathrm{BDCA} 1+\mathrm{mDC} \% \mathrm{MNC}$ & $0.38 \pm 0.18$ & $0.65 \pm 0.23$ & $<0.001^{\#}$ \\
\hline $\mathrm{BDCA} 3+\mathrm{mDC} \% \mathrm{MNC}$ & $0.17 \pm 0.04$ & $0.03 \pm 0.05$ & 0.374 \\
\hline Mono \%MNC & $15.56 \pm 9.24$ & $17.62 \pm 8.01$ & 0.473 \\
\hline CD14+ Mo \% MNC & $14.63 \pm 6.98$ & $16.98 \pm 7.00$ & 0.359 \\
\hline CD16+ Mo \% MNC & $3.07 \pm 2.19$ & $2.52 \pm 1.04$ & 0.448 \\
\hline CD16+ \%Mono & $15.58 \pm 7.86$ & $13.36 \pm 3.29$ & $0.020^{\#}$ \\
\hline NK \%MNC & $16.11 \pm 9.34$ & $10.90 \pm 4.91$ & 0.060 \\
\hline CD56hi NK \% MNC & $0.33 \pm 0.15$ & $0.29 \pm 0.19$ & 0.508 \\
\hline CD56lo NK \% MNC & $17.26 \pm 9.62$ & $10.36 \pm 5.33$ & $0.037^{\#}$ \\
\hline CD56hi \%NK & $2.54 \pm 2.05$ & $3.93 \pm 3.03$ & 0.106 \\
\hline MDSC \% CD33hi & $1.85 \pm 3.32$ & $3.42 \pm 7.74$ & 0.547 \\
\hline DR- $\% 14+$ APC & $0.51 \pm 1.55$ & $0.38 \pm 0.95$ & 0.800 \\
\hline DR- $\% 16+$ APC & $0.88 \pm 1.60$ & $0.44 \pm 0.75$ & 0.404 \\
\hline
\end{tabular}

*Independent $t$ test

${ }^{\#} P$ values with statistical significance were denoted 


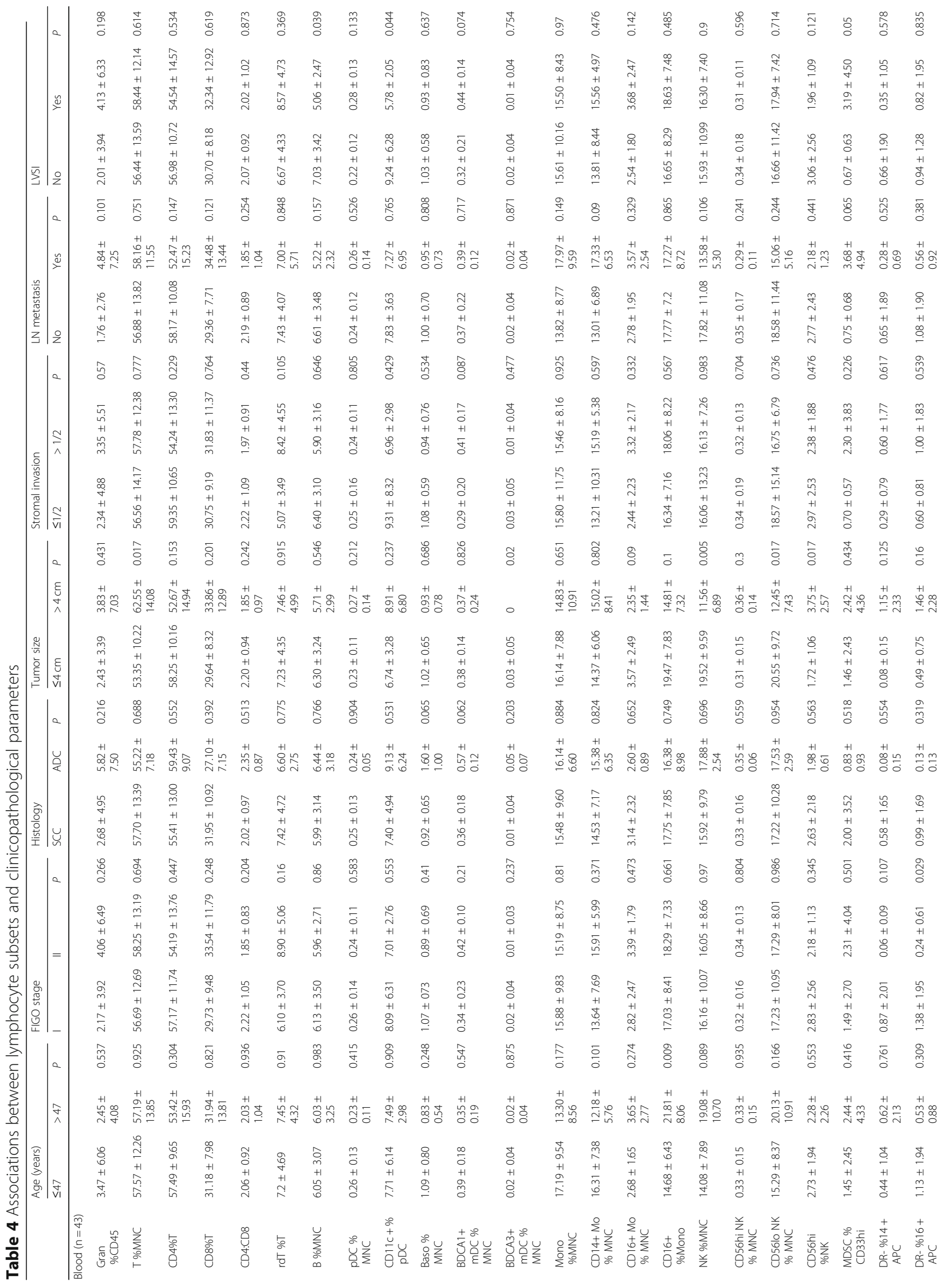




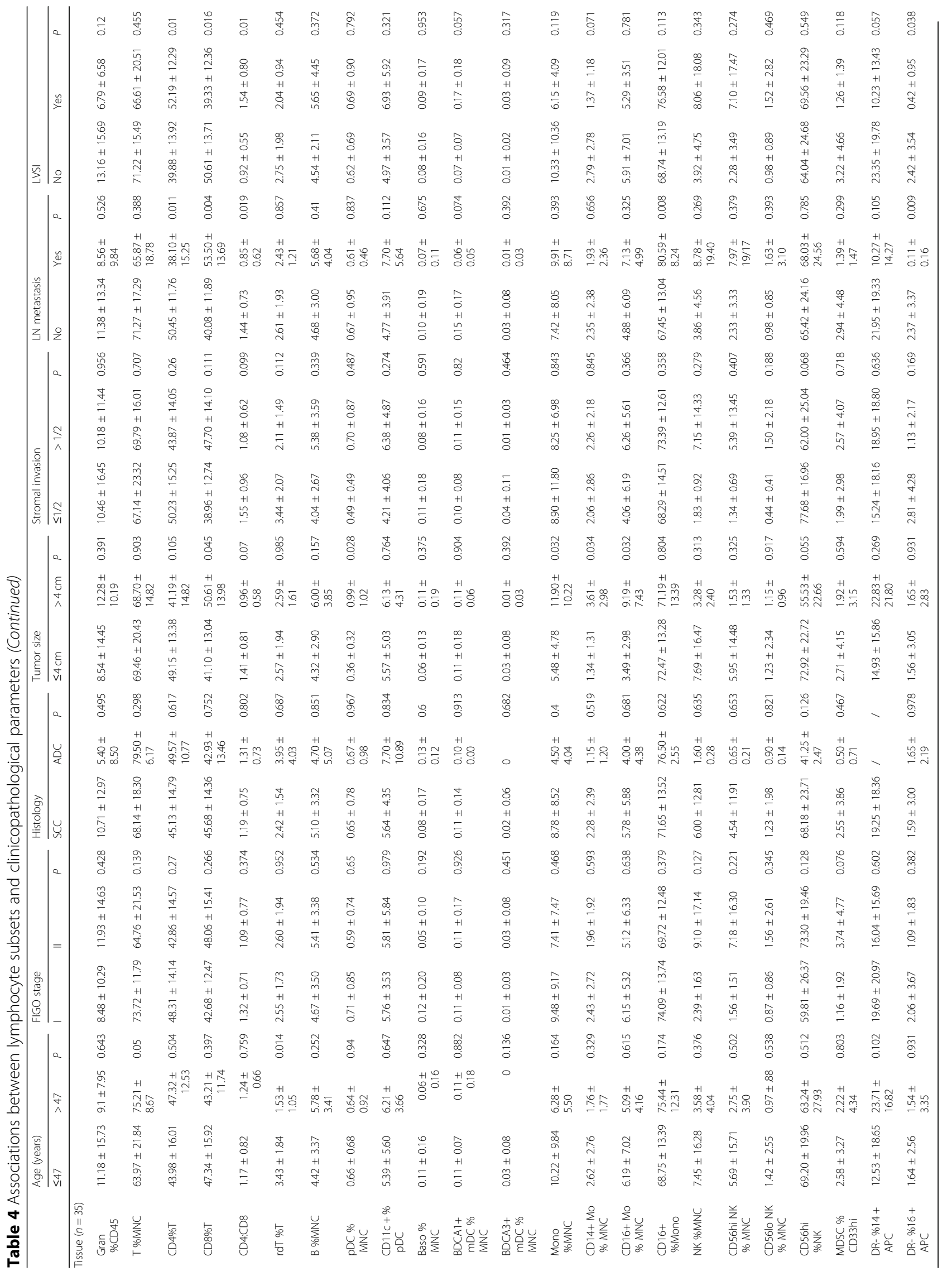


CD4+/CD8+ cell ratio $(p<0.05)$ (Table 4). In addition, no statistically significant differences were found in other lymphocyte subsets or clinicopathological variables.

\section{Prognostic implications of lymphocyte subpopulations The CD4+/CD8+ cell ratio differed significantly in both the peripheral blood and the tumor tissue}

We analyzed the correlations of 43 patients' lymphocyte subsets in the peripheral blood with basic characteristics, and the results revealed that most of the basic characteristics, such as age ( $\leq 47$ years vs. $>47$ years), stage (FIGO I vs. $\geq$ FIGO II), tumor size $(\leq 4 \mathrm{~cm}$ vs. $>4 \mathrm{~cm})$ and stromal invasion $(\leq 1 / 2$ vs. $>1 / 2)$, did not exhibit significant difference, but histology (SCC vs. ADC), LN metastasis status (negative vs. positive) and LVSI status (negative vs. positive) were found to be statistically significant ( $p<$ 0.05) (Table 5). When comparing the low level with the high level of a lymphocyte subset (the median value of the lymphocyte subset was used as the cut-off value), we found that most lymphocyte subsets did not exhibit significant differences $(p>0.05)$; however, the difference in the $\mathrm{CD} 4+/ \mathrm{CD} 8+$ cell ratio was significant $(p<0.05)$ (Table 5).

In addition, we analyzed the correlations of 35 patients' lymphocyte subsets in the tumor tissue with basic characteristics, and found that some basic characteristics, such as histology (SCC vs. ADC), tumor size $(\leq 4 \mathrm{~cm}$ vs. $>4 \mathrm{~cm}$ ), LN metastasis status (negative vs. positive) and LVSI status (negative vs. positive), exhibited significant difference $(p<0.05)$; however, age $(\leq 47$ years vs. $>$ 47 years), stage (FIGO I vs. $\geq$ FIGO II) and stromal invasion $(\leq 1 / 2$ vs. $>1 / 2)$ did not $(p>0.05)$ (Table 5$)$. The $\mathrm{CD} 8+$ cell proportion and $\mathrm{CD} 4+/ \mathrm{CD} 8+$ cell ratio were significantly different $(p<0.05)$, but significant differences were not found for other lymphocyte subsets $(p>$ 0.05) (Table 5).

\section{CD4+ cells, CD8+ cells and the CD4+/CD8+ cell ratio in the tumor tissue were closely related to recurrence}

We followed participants with a median follow-up time of 41 months and compared the distributions of different lymphocyte subsets between recurrent patients and disease-free patients. We found that the distributions of lymphocyte subsets were not significantly different between the recurrent patients and disease-free patients based on analysis of peripheral blood samples from 43 patients. However, in an analysis of tumor tissue samples from 35 patients, the $\mathrm{CD} 4+$ cell and $\mathrm{CD} 8+$ cell proportions and the CD4+/CD8+ cell ratio were significantly different between the recurrent patients and disease-free patients $(p<0.05)$, but there were no differences in other lymphocyte subsets (Table 6). The results indicated that the distributions of lymphocyte subsets in the peripheral
Table 5 Prognostic implication of lymphocyte subpopulations

\begin{tabular}{|c|c|c|}
\hline Parameters & $\begin{array}{l}P \text { value (Blood) } \\
n=43\end{array}$ & $\begin{array}{l}P \text { value (Tissue) } \\
n=35\end{array}$ \\
\hline Age ( $\leq 47$ years Vs. $>47$ years) & 0.755 & 0.979 \\
\hline Stage (FIGO I Vs. ZFIGO II) & 0.076 & 0.355 \\
\hline Histology (SCC Vs. ADC) & $0.002^{\#}$ & $0.002^{\#}$ \\
\hline Tumor $(\leq 4 \mathrm{~cm}$ Vs. $>4 \mathrm{~cm})$ & 0.214 & $0.032^{\#}$ \\
\hline Stromal invasion ( $\leq 1 / 2$ Vs. $>1 / 2)$ & 0.762 & 0.555 \\
\hline LN metastasis (negative Vs. positive) & $<0.001^{\#}$ & $0.001^{\#}$ \\
\hline LVSI (negative Vs. positive) & $0.017^{\#}$ & $0.034^{\#}$ \\
\hline Gran \%CD45 & 0.561 & 0.957 \\
\hline $\mathrm{T} \% \mathrm{MNC}$ & 0.413 & 0.939 \\
\hline $\mathrm{CD} 4 \% \mathrm{~T}$ & 0.066 & 0.090 \\
\hline $\mathrm{CD} 8 \% \mathrm{~T}$ & 0.088 & $0.005^{\#}$ \\
\hline CD4:CD8 & $0.048^{\#}$ & $0.008^{\#}$ \\
\hline rdT \% $\%$ & 0.294 & 0.317 \\
\hline $\mathrm{B} \% \mathrm{MNC}$ & 0.544 & 0.513 \\
\hline $\mathrm{pDC} \% \mathrm{MNC}$ & 0.727 & 0.278 \\
\hline $\mathrm{CD} 11 \mathrm{c}+\% \mathrm{pDC}$ & 0.576 & 0.509 \\
\hline Baso \% MNC & 0.528 & 0.591 \\
\hline $\mathrm{BDCA} 1+\mathrm{mDC} \% \mathrm{MNC}$ & 0.337 & 0.132 \\
\hline $\mathrm{BDCA} 3+\mathrm{mDC} \% \mathrm{MNC}$ & 0.632 & 0.371 \\
\hline Mono \%MNC & 0.808 & 0.812 \\
\hline CD14+ Mo \% MNC & 0.961 & 0.481 \\
\hline CD16+ Mo \% MNC & 0.576 & 0.062 \\
\hline CD16+ \%Mono & 0.576 & 0.062 \\
\hline NK \%MNC & 0.752 & 0.533 \\
\hline CD56hi NK \% MNC & 0.820 & 0.648 \\
\hline CD56lo NK \% MNC & 0.525 & 0.481 \\
\hline CD56hi \%NK & 0.525 & 0.563 \\
\hline MDSC \% CD33hi & 0.576 & 0.679 \\
\hline DR- $\% 14+$ APC & 0.443 & 0.536 \\
\hline DR- $\% 16+A P C$ & 0.093 & 0.106 \\
\hline
\end{tabular}

*Median value of lymphocyte was used as cut-off value

${ }^{\#} P$ values with statistical significance were denoted

blood may not be the optimal method for detecting recurrence, while the detection of lymphocyte subsets in tumor tissue may be a possible strategy for detecting recurrence.

\section{Discussion}

Previous studies have widely noted that the distributions of immunological parameters are changed in patients with cervical cancer and possibly associated with dysfunction in the antitumor immune system [5, 17-20]. The presence of tumor infiltrating lymphocytes that mediate the antitumor immune response has been observed in several types of cancer, including ovarian cancer, 
Table 6 Lymphocyte subpopulations: recurrent patients vs. disease-free patients

\begin{tabular}{|c|c|c|c|c|c|c|}
\hline & Blood $(n=43)$ & & & Tissue $(n=35)$ & & \\
\hline & Non-recurrent & Recurrent & $P$ & Non-recurrent & Recurrent & $P$ \\
\hline Gran \%CD45 & $2.22 \pm 4.38$ & $6.65 \pm 7.52$ & 0.147 & $10.66 \pm 13.45$ & $8.28 \pm 8.24$ & 0.682 \\
\hline T \%MNC & $57.32 \pm 13.27$ & $57.83 \pm 11.21$ & 0.921 & $68.67 \pm 19.01$ & $71.23 \pm 11.52$ & 0.754 \\
\hline $\mathrm{CD} 4 \% \mathrm{~T}$ & $57.45 \pm 10.67$ & $48.50 \pm 18.24$ & 0.071 & $48.07 \pm 14.41$ & $33.12 \pm 13.59$ & $0.018^{\#}$ \\
\hline CD8\% & $30.38 \pm 8.59$ & $36.41 \pm 17.03$ & 0.151 & $42.87 \pm 13.46$ & $57.92 \pm 10.76$ & $0.015^{\#}$ \\
\hline CD4:CD8 & $2.14 \pm 0.97$ & $1.65 \pm 0.85$ & 0.196 & $1.32 \pm 0.75$ & $0.62 \pm 0.34$ & $0.032^{\#}$ \\
\hline rdT \% & $7.21 \pm 4.54$ & 9.30 & 0.657 & $2.73 \pm 2.00$ & $2.22 \pm 1.23$ & 0.571 \\
\hline B $\% \mathrm{MNC}$ & $6.13 \pm 3.31$ & $5.61 \pm 1.93$ & 0.693 & $5.36 \pm 3.62$ & $3.65 \pm 1.78$ & 0.270 \\
\hline $\mathrm{pDC} \% \mathrm{MNC}$ & $0.24 \pm 0.12$ & $0.26 \pm 0.16$ & 0.696 & $0.69 \pm 0.84$ & $0.47 \pm 0.41$ & 0.541 \\
\hline$C D 11 c+\% p D C$ & $8.05 \pm 5.19$ & $4.63 \pm 2.41$ & 0.208 & $5.92 \pm 4.90$ & $5.27 \pm 4.20$ & 0.769 \\
\hline Baso \% MNC & $0.89 \pm 0.63$ & $1.36 \pm 0.91$ & 0.091 & $0.10 \pm 0.17$ & $0.03 \pm 0.08$ & 0.390 \\
\hline $\mathrm{BDCA} 1+\mathrm{mDC} \% \mathrm{MNC}$ & $0.37 \pm 0.19$ & $0.43 \pm 0.10$ & 0.595 & $0.12 \pm 0.15$ & $0.07 \pm 0.05$ & 0.382 \\
\hline $\mathrm{BDCA} 3+\mathrm{mDC} \% \mathrm{MNC}$ & $0.02 \pm 0.04$ & 0 & $0.022^{\#}$ & $0.02 \pm 0.07$ & .0000 & 0.441 \\
\hline Mono \% MNC & $14.97 \pm 9.08$ & $18.13 \pm 10.17$ & 0.391 & $7.89 \pm 7.83$ & $10.98 \pm 10.67$ & 0.413 \\
\hline CD14+ Mo \% MNC & $14.35 \pm 7.29$ & $16.63 \pm 4.44$ & 0.550 & $1.90 \pm 2.03$ & $3.37 \pm 3.23$ & 0.175 \\
\hline CD16+ Mo \% MNC & $2.91 \pm 1.89$ & $4.20 \pm 3.91$ & 0.560 & $5.14 \pm 5.30$ & $7.62 \pm 7.46$ & 0.357 \\
\hline CD16+ \%Mono & $17.40 \pm 7.24$ & $18.80 \pm 12.82$ & 0.745 & $72.16 \pm 13.58$ & $71.30 \pm 12.18$ & 0.889 \\
\hline NK \%MNC & $16.85 \pm 9.95$ & $12.37 \pm 4.01$ & 0.251 & $6.12 \pm 13.48$ & $3.98 \pm 6.32$ & 0.709 \\
\hline CD56hi NK \% MNC & $0.33 \pm 0.15$ & $0.30 \pm 0.12$ & 0.693 & $4.50 \pm 12.69$ & $3.40 \pm 5.76$ & 0.839 \\
\hline CD56lo NK \% MNC & $17.68 \pm 10.17$ & $14.35 \pm 3.59$ & 0.527 & $1.37 \pm 2.10$ & $0.55 \pm 0.60$ & 0.357 \\
\hline CD56hi \%NK & $2.61 \pm 2.19$ & $2.05 \pm 0.58$ & 0.616 & $64.10 \pm 24.34$ & $74.82 \pm 21.91$ & 0.337 \\
\hline MDSC \% CD33hi & $1.53 \pm 2.84$ & $4.08 \pm 5.80$ & 0.155 & $2.67 \pm 4.16$ & $1.42 \pm 1.30$ & 0.479 \\
\hline DR- $\% 14+$ APC & $0.58 \pm 1.65$ & $0.08 \pm 0.15$ & 0.554 & $16.21 \pm 17.23$ & $24.48 \pm 22.78$ & 0.335 \\
\hline DR- $\% 16+$ APC & $1.00 \pm 1.68$ & $0.05 \pm 0.06$ & 0.272 & $1.29 \pm 2.18$ & $2.75 \pm 4.98$ & 0.284 \\
\hline
\end{tabular}

${ }^{\#} P$ values with statistical significance were denoted

breast cancer and pancreatic cancer, and is closely correlated with prognosis [21-23]. However, most previous studies have focused on the distributions of lymphocyte subsets in the peripheral blood, and few studies have revealed the distributions of lymphocyte subpopulations in tumor tissue. Obviously, the lymphocyte subsets in tumor tissue are more representative of the tumor microenvironment than those in the peripheral blood. Therefore, our study evaluated lymphocyte subsets in tumor tissue and peripheral blood samples from cervical cancer patients and precancerous lesion patients. We found inconsistencies in the distributions of lymphocyte subsets between the tumor tissue and peripheral blood samples from the cervical cancer patients and precancerous lesion patients, and the results suggested an imbalance in the homeostasis of the host immune system in the patients with cervical cancer.

The primary result of this study is that total $\mathrm{T}$ lymphocyte, CD8+ T cell and granulocyte proportions were significantly higher in the tumor tissue than in the peripheral blood, while $\mathrm{CD} 4+\mathrm{T}$ cell and $\mathrm{rdT}$ cell proportions and the $\mathrm{CD} 4+/ \mathrm{CD} 8+$ cell ratio were significantly lower in the tumor tissue than in the peripheral blood from cervical cancer patients. However, when compared with the peripheral blood samples from precancerous lesion patients, those from cervical cancer patients did not exhibit significant differences in lymphocyte subsets. Our results indicated that $\mathrm{T}$ cellmediated immunity was impaired in patients with cervical cancer, which was more distinct in the tumor tissue microenvironment than in the peripheral blood. In addition, our previous study found that a population of highly activated, immunosuppressive HLADR ${ }^{\text {hi }}$ Tregs in cervical squamous cell carcinoma and a high frequency of stomal HLADR ${ }^{\text {hi }}$ Tregs in patients were significantly associated with relatively poor prognosis [24]. Tregs have fundamental roles in the establishment and maintenance of the peripheral immune tolerance microenvironment [25]. Future research should be conducted to clarify the relationship between HPV infection and Tregs in the tumor tissue and peripheral blood of cervical cancer patients. Increasing granulocyte numbers have been reported to be associated with tumor growth and angiogenesis. A study by Zheng et al. reported that 
granulocytes but not monocytes significantly promoted tumor growth, implying that granulocytes play key roles in tumor growth and angiogenesis [26]. These findings support the results obtained in our study that the granulocyte proportion was significantly higher in tumor tissue than in the peripheral blood. In addition, a study collected tumor tissue and peripheral blood samples from patients with cervical cancer and found that the CD4+ cell proportion and CD4+/CD8+ cell ratio were significantly lower in cervical cancer tissue than in the peripheral blood, which is consistent with our results [21]. However, the limitation of the previous study was that they had not evaluated lymphocyte subsets other than $\mathrm{CD} 4+$ and $\mathrm{CD} 8+$ cells; thus, they could not make any interpretations about the correlations of lymphocyte subsets with clinicopathologic parameters or prognostic significance.

Furthermore, our study revealed that the pDCs cell proportion was significantly increased in tumor tissue compared with the peripheral blood, but the BDCA1+ $\mathrm{mDCs}$ proportion was significantly decreased. No significant differences were found in $\mathrm{CD} 11 \mathrm{c}+\mathrm{pDCs}$ or $\mathrm{BDCA} 3+\mathrm{mDCs}$ between the tumor tissue and the peripheral blood. In addition, total pDC and BDCA1+ $\mathrm{mDC}$ proportions in the peripheral blood were significantly lower in cervical cancer patients than in precancerous lesion patients. Dysfunction of DCs induced by tumors can be an important mechanism underlying tumorinduced immune escape because DCs are one of the key types of professional antigen-presenting cells (APCs) that induce tumor-specific immune responses, particularly by cross-priming through MHC class I antigen presentation [27]. Professional APCs are one of the most important inducers of antigen-specific immune responses, and their potentially defective function causes a strong impairment in immunosurveillance in tumorbearing hosts [28]. The alterations in host lymphocyte subsets that occur in the early stage of tumor progression, including precancerous lesions, may provide some directions to evaluate the roles of different lymphocyte subpopulations in the transition of premalignant lesions into invasive cancer [29].

Our study found that the levels of total monocytes and CD14+ monocytes were significantly decreased in tumor tissue compared to the peripheral blood, while the level of CD16+ monocyte was significantly elevated. The CD16+ monocyte proportion was also significantly increased in the peripheral blood of cervical cancer patients compared to that of precancerous lesion patients. A study by Anna et al. assessed CD14+/CD16+ monocytes in human blood and showed that CD14+/CD16+ monocytes constitute the main subset of blood monocytes involved in the antitumor response and function by producing proinflammatory cytokines and reactive nitrogen and exhibiting increased cytotoxic/cytostatic activity [30]. Therefore, our study revealed that the antitumor response of the host may be defective due to the decreases in total monocyte and CD14+ monocyte levels in tumor tissue.

Our study showed that NK cell and CD56 ${ }^{\text {low }}$ NK cell proportions were significantly decreased in tumor tissue compared with the peripheral blood, while the percentage of CD56 $6^{\text {high }}$ NK cells was significantly higher in tumor tissue. NK cells are the component of the innate immune surveillance system that plays an important role in host defense immunity. NK cells may be important for the regional immune reaction against cervical carcinoma [31]; thus, the decreases in NK cell subsets in tumor tissue are possibly related to disease progression.

Alterations in the distributions of lymphocyte subsets can occur in the peripheral blood, lymph nodes and other sites in cancer patients, which would induce immune suppression that varies depending on tumor etiology, location, histological type and clinical stage [29]. We detected the associations of lymphocyte subsets with clinicopathological parameters and found that BDCA3+ $\mathrm{mDC}$, total NK cell and CD56 ${ }^{\text {low }} \mathrm{NK}$ cell proportions in the peripheral blood were significantly lower in the tumor size $>4 \mathrm{~cm}$ group than in the tumor size $\leq 4 \mathrm{~cm}$ group. In contrast, CD8+ cell, pDC, total monocyte, CD14+ monocyte and CD 16+ monocyte proportions in tumor tissue were significantly higher in the tumor size $>4 \mathrm{~cm}$ group than in the tumor size $\leq 4 \mathrm{~cm}$ group. In addition, the $\mathrm{CD} 11 \mathrm{c}+\mathrm{pDC}$ proportion in the peripheral blood was significantly lower in LVSI-positive patients than in LVSI-negative patients, and LVSI was correlated with a significantly reduced CD8+ cell proportion in tumor tissue. The CD4+ cell proportion and $\mathrm{CD} 4+1$ $\mathrm{CD} 8+$ cell ratio in tumor tissue were significantly reduced. These results indicated some inconsistent implications about clinicopathological parameters between the peripheral blood and tumor tissue. Nevertheless, our results implied that there were close associations between lymphocyte subsets and clinicopathological parameters and that the host immunity system was affected by differences in age, tumor size, LVSI and LN metastasis in cervical cancer patients. Further research should be carried out to clearly reveal the associations between lymphocyte subsets and clinicopathological parameters.

We divided the lymphocyte levels into a low-level group and high-level group by cut-off values defined as the median value for the indicated lymphocyte subset. The results revealed that the $\mathrm{CD} 4+/ \mathrm{CD} 8+$ cell ratio was significantly different in both the peripheral blood and tumor tissue when the low level was compared with the high level of the lymphocyte subsets. Mounting evidence has shown that the innate mechanisms that maintain immune cell homeostasis and control the magnitude of 
immune responses can undergo dramatic dysregulation, which leads to suppression of the immune system and enables tumors to escape immune defense because of the influence of tumor cells [29]. After a median followup time of 41 months, we found that the distributions of lymphocyte subsets in the peripheral blood were not significantly different between recurrent patients and disease-free patients. Interestingly, the $\mathrm{CD} 4+$ cell proportion and $\mathrm{CD} 4+/ \mathrm{CD} 8+$ cell ratio in tumor tissue were significantly decreased in the recurrent patients compared with the disease-free patients, while the CD8+ cell proportion was significantly increased. Our results are similar to those of a previous study by Sheu et al., which reported that a decreased proportion of tumorinfiltrating $\mathrm{CD} 4+\mathrm{T}$ cells and a reduced $\mathrm{CD} 4+/ \mathrm{CD} 8+$ cell ratio were highly correlated with rapid tumor growth and lymph node metastasis due to a poor antitumor response in cervical cancer [31]. A previous study showed that decreased proportions of tumor-infiltrating CD4+ T cells and a reversed CD4+/CD8+ cell ratio were significantly associated with the clinical outcome of patients with cervical cancer [32]. As is well acknowledged, the development of invasive cervical tumors from cervical intraepithelial neoplasia (CIN) is always accompanied by HPV infection [33]. Persistent infection with high-risk HPV in CIN patients may generate an immunosuppressive microenvironment that allows progression to cervical cancer [34]. A direct correlation between HPV infection and the CD4+/CD8+ cell ratio has been reported [35]. A study detected populations of $\mathrm{T}$ cells (CD4+ and CD8+ cells) in high-risk HPV-infected precancerous and cancerous lesions of the uterine cervix and found that CD8+ $\mathrm{T}$ cell numbers far exceeded CD4+ $\mathrm{T}$ cell numbers in invasive cancers [8]. CD4+ and $\mathrm{CD} 8+\mathrm{T}$ cells have important roles in the natural history of HPV-infected cervical cancer [8].

Our study provides some information about the distributions of lymphocyte subsets in the peripheral blood, tumor tissue and precancerous lesions and reveals the correlations of lymphocyte subsets with clinical characteristics and prognostic variables. Nonetheless, some limitations of our study should be stated, and we should interpret our results with caution. First, some of the analyses could not identify significant differences, which may be due to the lack of a large sample size. We would like to enlarge our sample of participants in future research. In addition, we could not perform subgroup analyses according to different histological types, clinical stages or HPV statuses due to the small sample size. Last, we could not generate survivorship curves according to different distributions of lymphocyte subsets because of the short follow-up time. We will continue to follow our participants and conduct survival analysis in future studies. To provide more knowledge about the distributions of lymphocyte subpopulations in patients with cervical cancer and their relationships with prognosis, a prospective study should be performed.

\section{Conclusions}

Taken together, our results identified significant alterations in lymphocyte subsets in tumor tissue and elucidated that host antitumor immunity may be suppressed. Total T cells, CD4+ cells, CD8+ cells, the CD4+/CD8+ cell ratio, DCs and monocytes in tumor tissue may have significant roles in predicting the homeostasis of the host antitumor immune response. Alterations in lymphocyte subsets were closely correlated with clinicopathologic and prognostic parameters in cervical cancer patients. Further research will lead to a better understanding of the clinical significance of lymphocyte subsets in cervical cancer.

\section{Supplementary information}

Supplementary information accompanies this paper at https://doi.org/10. 1186/s12885-020-6633-X.

\section{Additional file 1}

\section{Abbreviations}

Baso: Basophilic Granulocytes; CIN: Cervical Intraepithelial Neoplasia; DCs: Dendritic cells; HIV: Human Immunodeficiency Virus; HPV: Human Papillomavirus; LVSI: Lymph Vascular Space Invasion; mDCs: Myeloid Dendritic Cells; MDSCs: Myeloid-Derived Suppressor Cells; NK: Natural Killer cells; PBMCs: Peripheral Blood Mononuclear Cells; pDCs: Plasmacytoid Dendritic Cells; PFS: Progression-free Survival

\section{Acknowledgments}

Not applicable.

\section{Authors' contributions}

YW, SY, SG, XP, LX, XZ and HY contributed to the contraception and design of the study. YW, and SY collected and analyzed patients' clinicopathological data. SG and XZ performed and analyzed the flow cytometry. YW, SY, XP, LX and $H Y$ were major contributors in writing the manuscript. YW, SY, SG, XP, $L X, X Z$ and $H Y$ read and approved the final manuscript.

\section{Funding}

The study was supported by grants from National Natural Science Foundation of China (81702558) and Fudan University Shanghai Cancer Center (YJ201603). The funding bodies didn't participate in the design of the study and collection, analysis, and interpretation of data and in writing the manuscript.

\section{Availability of data and materials}

The dataset supporting the conclusions of this article is available upon request. Please contact Prof. Huijuan Yang (huijuanyang@hotmail.com).

\section{Ethics approval and consent to participate}

The study was approved by the Fudan University Shanghai Cancer Center review board and the requirement for written informed consent was waived due to its retrospective design.

\section{Consent for publication}

Not applicable. This manuscript does not contain any individual person data.

Competing interests

The authors declare that they have no competing interests. 


\section{Author details}

${ }^{1}$ Department of Gynecological Oncology, Fudan University Shanghai Cancer Center, Fudan University, Shanghai, China. ${ }^{2}$ Department of Oncology, Shanghai Medical College, Fudan University, Shanghai 200032, China. ${ }^{3}$ Unit of Innate Defense and Immune Modulation, Key Laboratory of Molecular Virology and Immunology, Institute Pasteur of Shanghai, Chinese Academy of Sciences, Shanghai, China.

\section{Received: 6 September 2019 Accepted: 14 February 2020}

Published online: 04 March 2020

\section{References}

1. Chen W, Zheng R, Baade PD, Zhang S, Zeng H, Bray F, Jemal A, Yu XQ, He J. Cancer statistics in China, 2015. CA Cancer J Clin. 2016;66(2):115-32.

2. Nobbenhuis MA, Walboomers JM, Helmerhorst TJ, Rozendaal L, Remmink AJ, Risse EK, van der Linden HC, Voorhorst FJ, Kenemans P, Meijer CJ. Relation of human papillomavirus status to cervical lesions and consequences for cervical-cancer screening: a prospective study. Lancet (London, England). 1999;354(9172):20-5.

3. Bosch FX, Manos MM, Munoz N, Sherman M, Jansen AM, Peto J, Schiffman $\mathrm{MH}$, Moreno V, Kurman R, Shah KV. Prevalence of human papillomavirus in cervical cancer: a worldwide perspective. International biological study on cervical cancer (IBSCC) study group. J Natl Cancer Inst. 1995;87(11):796-802.

4. Mehta AM, Mooij M, Brankovic I, Ouburg S, Morre SA, Jordanova ES. Cervical carcinogenesis and immune response gene polymorphisms: a review. J Immunol Res. 2017:2017:8913860.

5. Das S, Karim S, Datta Ray C, Maiti AK, Ghosh SK, Chaudhury K. Peripheral blood lymphocyte subpopulations in patients with cervical cancer. Int J Gynaecol Obstet. 2007;98(2):143-6.

6. Bachtiary B, Herbacek I, Zideck T, Knocke TH, Dimopoulos J, Poetter R, Micksche M. Impact of radiotherapy with and without concurrent cisplatin on lymphocyte subpopulations in cervical cancer patients. Anticancer Res. 2005;25(6c):4673-8.

7. Steele JC, Mann CH, Rookes S, Rollason T, Murphy D, Freeth MG, Gallimore PH, Roberts S. T-cell responses to human papillomavirus type 16 among women with different grades of cervical neoplasia. Br J Cancer. 2005;93(2):248-59.

8. Monnier-Benoit S, Mauny F, Riethmuller D, Guerrini JS, Capilna M, Felix S, Seilles E, Mougin C, Pretet JL. Immunohistochemical analysis of CD4+ and CD8+ T-cell subsets in high risk human papillomavirus-associated premalignant and malignant lesions of the uterine cervix. Gynecol Oncol. 2006; 102(1):22-31.

9. Molling JW, de Gruij TD, Glim J, Moreno M, Rozendaal L, Meijer CJ, van den Eertwegh AJ, Scheper RJ, von Blomberg ME, Bontkes HJ. CD4(+)CD25hi regulatory $\mathrm{T}$-cell frequency correlates with persistence of human papillomavirus type 16 and $T$ helper cell responses in patients with cervical intraepithelial neoplasia. Int J Cancer. 2007;121(8):1749-55.

10. Nedergaard BS, Ladekarl M, Thomsen HF, Nyengaard JR, Nielsen K. Low density of $\mathrm{CD} 3+, \mathrm{CD} 4+$ and CD8+ cells is associated with increased risk of relapse in squamous cell cervical cancer. Br J Cancer. 2007;97(8):1135-8.

11. Piersma SJ, Jordanova ES, van Poelgeest MI, Kwappenberg KM, van der Hulst JM, Drijfhout JW, Melief CJ, Kenter GG, Fleuren GJ, Offringa R, et al. High number of intraepithelial CD8+ tumor-infiltrating lymphocytes is associated with the absence of lymph node metastases in patients with large early-stage cervical cancer. Cancer Res. 2007;67(1):354-61.

12. Woo YL, Sterling J, Damay I, Coleman N, Crawford R, van der Burg SH, Stanley M. Characterising the local immune responses in cervical intraepithelial neoplasia: a cross-sectional and longitudinal analysis. BJOG. 2008;115(13):1616-21 discussion 1621-1612.

13. de Vos van Steenwijk PJ, Heusinkveld M, Ramwadhdoebe TH, Lowik MJ, van der Hulst JM, Goedemans R, Piersma SJ, Kenter GG, van der Burg SH. An unexpectedly large polyclonal repertoire of HPV-specific T cells is poised for action in patients with cervical cancer. Cancer Res. 2010;70(7):2707-17.

14. Ye F, Yu Y, Hu Y, Lu W, Xie X. Alterations of dendritic cell subsets in the peripheral circulation of patients with cervical carcinoma. J Exp Clin Cancer Res. 2010;29:78.

15. Chang WC, Li CH, Chu LH, Huang PS, Sheu BC, Huang SC. Regulatory T cells suppress natural killer cell immunity in patients with human cervical carcinoma. Int J Gynecol Cancer. 2016;26(1):156-62.

16. Jingwen Wu BP, Yang W, Wu X, Zhang X, Yang H. Measurement of CD4+ CD25highCD127low regulatory $T$ cells in peripheral blood from cervical cancers and precursors. China Oncology. 2013;23(3):167-72.
17. Alsabti EA. The immunostatus of untreated cervical carcinoma. Gynecol Oncol. 1980;9(1):6-11.

18. Ishiguro T, Sugitachi I, Katoh K. T and B lymphocytes in patients with squamous cell carcinoma of the uterine cervix. Gynecol Oncol. 1980;9(1):80-5.

19. Jain R, Gupta MM, Parashari A, Kaur S, Luthra UK. Peripheral blood lymphocyte subpopulations in Indian women with cervical intraepithelial neoplasia and invasive cancer--an immunocytochemical study using monoclonal antibodies. Cancer Lett. 1990;54(1-2):17-20.

20. Saxena VK, Chander S, Chandra M, Sachdeva S, Ghosh D, Saxena HM. A study of T lymphocyte population in cancer of the cervix. Effects of pelvic irradiation. Indian J Cancer. 1985;22(3):173-7.

21. Das D, Sarkar B, Mukhopadhyay S, Banerjee C, Biswas Mondal S. An altered ratio of CD4+ and CD8+ T lymphocytes in cervical Cancer tissues and peripheral blood - a prognostic clue? Asian Pac J Cancer Prev. 2018;19(2):471-8.

22. Xu YF, Lu Y, Cheng H, Shi S, Xu J, Long J, Liu L, Liu C, Yu X. Abnormal distribution of peripheral lymphocyte subsets induced by PDAC modulates overall survival. Pancreatology : official journal of the International Association of Pancreatology (IAP) [et al]. 2014;14(4):295-301.

23. Lee KH, Kim EY, Yun JS, Park YL, Do SI, Chae SW, Park CH. The prognostic and predictive value of tumor-infiltrating lymphocytes and hematologic parameters in patients with breast cancer. BMC Cancer. 2018;18(1):938.

24. Yang H, Ye S, Goswami S, Li T, Wu J, Cao C, Ma J, Lu B, Pei X, Chen Y, et al. Highly immunosuppressive HLADR (hi) regulatory $T$ cells are associated with unfavorable outcomes in cervical squamous cell carcinoma. Int J Cancer. 2020;146(7):1993-2006

25. Allan SE, Broady R, Gregori S, Himmel ME, Locke N, Roncarolo MG, Bacchetta R, Levings MK. CD4+ T-regulatory cells: toward therapy for human diseases. Immunol Rev. 2008:223:391-421.

26. Zheng Q, Li X, Cheng X, Cui T, Zhuo Y, Ma W, Zhao X, Zhao P, Liu X, Feng W. Granulocyte-macrophage colony-stimulating factor increases tumor growth and angiogenesis directly by promoting endothelial cell function and indirectly by enhancing the mobilization and recruitment of proangiogenic granulocytes. Tumour Biol. 2017;39(2):1010428317692232.

27. Dominguez D, Ye C, Geng Z, Chen S, Fan J, Qin L, Long A, Wang L, Zhang Z, Zhang Y, et al. Exogenous IL-33 Restores Dendritic Cell Activation and Maturation in Established Cancer. J Immunol (Baltimore, Md : 1950). 2017;198(3):1365-75.

28. Feng T, Zhou JH, Liu J, Ye F, Lu W, Xie X. Altered expression levels of HLA class and costimulatory molecules on circulating monocytes from patients with cervical intraepithelial neoplasia and squamous cervical cancer. Mol Med Rep. 2012;6(6):1301-4.

29. Kurmyshkina OV, Kovchur PI, Schegoleva LV, Volkova TO. T- and NK-cell populations with regulatory phenotype and markers of apoptosis in circulating lymphocytes of patients with CIN3 or microcarcinoma of the cervix: evidence for potential mechanisms of immune suppression. Infect Agents Cancer. 2017;12:56.

30. Szaflarska A, Baj-Krzyworzeka M, Siedlar M, Weglarczyk K, Ruggiero I, Hajto B, Zembala M. Antitumor response of CD14+/CD16+ monocyte subpopulation. Exp Hematol. 2004;32(8):748-55.

31. Sheu BC, Hsu SM, Ho HN, Lin RH, Torng PL, Huang SC. Reversed CD4/CD8 ratios of tumor-infiltrating lymphocytes are correlated with the progression of human cervical carcinoma. Cancer. 1999;86(8):1537-43.

32. Shah $W$, Yan $X$, Jing $L$, Zhou $Y$, Chen $H$, Wang $Y$. A reversed CD4/CD8 ratio of tumor-infiltrating lymphocytes and a high percentage of CD4(+)FOXP3(+ ) regulatory $T$ cells are significantly associated with clinical outcome in squamous cell carcinoma of the cervix. Cel Mol Immunol. 2011;8(1):59-66.

33. Monroy-Garcia A, Gomez-Lim MA, Weiss-Steider B, Hernandez-Montes J, Huerta-Yepez S, Rangel-Santiago JF, Santiago-Osorio E, Mora Garcia Mde L. Immunization with an HPV-16 L1-based chimeric virus-like particle containing HPV-16 E6 and E7 epitopes elicits long-lasting prophylactic and therapeutic efficacy in an HPV-16 tumor mice model. Arch Virol. 2014;159(2):291-305.

34. de Lourdes M-GM, Lopez-Cisneros S, Gutierrez-Serrano V, Garcia-Rocha R, Weiss-Steider B, Hernandez-Montes J. HPV-16 infection is associated with a high content of CD39 and CD73 Ectonucleotidases in cervical samples from patients with CIN-1. Mediat Inflamm. 2019;2019:4651627.

35. Zhang D, Tang WJ, Tang D, Zhou J, Chou L, Tao L, Lu LM. The ratio of CD4/CD8 T-cells in human papillomavirus-positive laryngeal squamous cell carcinoma accounts for improved outcome. Acta Otolaryngol. 2016;136(8):826-33.

\section{Publisher's Note}

Springer Nature remains neutral with regard to jurisdictional claims in published maps and institutional affiliations. 